\title{
COHERENT INTERACTION OF THE RELATIVISTIC ELECTRON BEAM WITH THE PLASMA AND HIGH-GRADIENT ACCELERATING FIELDS GENERATION
}

\author{
G. Oksuzyan ${ }^{\dagger}$, M. Ivanyan, A. Vardanyan, YerPhI, Yerevan, Armenia
}

\begin{abstract}
The results of the experimental investigations of the relativistic electron bunched-beam interaction with plasma are presented. Energy spectrum of the electron beam on the exit of the interaction chamber at energy 50 and $20 \mathrm{MeV}$ was measured. Coherent interaction of the electron beam with ultra-high frequency (UHF) plasma is investigated and indicated that in coherent conditions the greate fields may be generated, that are driven to the sharply beam current dependence on the phase of the UHF wave. Discovered new coherent effect may be used for development of an accelerating module with high gradient. The effect may be amplified with the help of powerful parametric resonance. It may be useful for thermonuclear researches, where the plasma is heated up by a high current relativistic electron beam as well.
\end{abstract}

\section{RESULTS}

At present, plasma methods for the acceleration of charged particles are being actively developed (see, e.g., [1], the reviews by Ya.B. Fainberg [2--4], and the literature cited therein). Theoretical and experimental papers aimed at investigating the mechanisms for the generation of strong wakefields show that one of the main directions in this area - the wakefield excitation by relativistic electron bunches - has many useful applications (see, e.g., [5--11]), in particular, in creating the conditions for the coherent interaction of a relativistic electron beam with a plasma. These conditions can be achieved, e.g., in the interaction of a modulated electron beam with a plasma driven by a microwave pulse at the frequency of the accelerating field in an accelerator. We carried out experiments with a decaying plasma and a microwave-driven plasma. The experimental device consists of a four stage linear accelerator (linac), plasma chamber, magnetic analyzers, and other elements shown in Fig. 1. The parameters of the electron beam are as follows: the peak value of the beam current is $0.1--0.15$ A, the modulation frequency is $f_{m}=2797.3 \mathrm{MHz}$, the beam diameter is about $1 \mathrm{~cm}$, and the length of electron bunches is about $1 \mathrm{~cm}$. The plasma chamber is an 8 -cm-diameter glass tube with the electrodes separated by a distance of about $40 \mathrm{~cm}$. The plasma is created by applying a dc voltage to the hollow electrodes of the discharge chamber through a high-voltage modulator, which is switched on synchronously with the linac operating in the pulsed mode. During the pulse, the peak plasma current is 100--150 A. The plasma density is measured both with a radio interferometer at a wavelength of $3 \mathrm{~cm}$ and from the cutoff of the probing signal. The optimum plasma density is chosen by delaying the current pulse of an electron beam with respect to the plasma current pulse. Our experiments were conducted in the absence of an external magnetic field. We begin by describing experiments with a decaying plasma. The electron energy spectra were measured over times much longer than the repetition period of the current pulses; i.e., we measured the integral spectra. In order to improve the measurement accuracy, a magnetic analyzer installed behind the interaction chamber was equipped with an additional solenoid (23) (Fig. 1) in which the number of coils was two orders of magnitude smaller than that in the main magnet. Both the magnets and solenoids were powered by a dc source with an instability of about $0.03 \%$, the instability of the voltage source that supplied the linac being about $0.1 \%$. The current of the electrons that traversed the entire plasma region was measured with an accuracy of about $10 \%$, the accuracy of the measurements of the electron energybeing about $0.3 \%$.

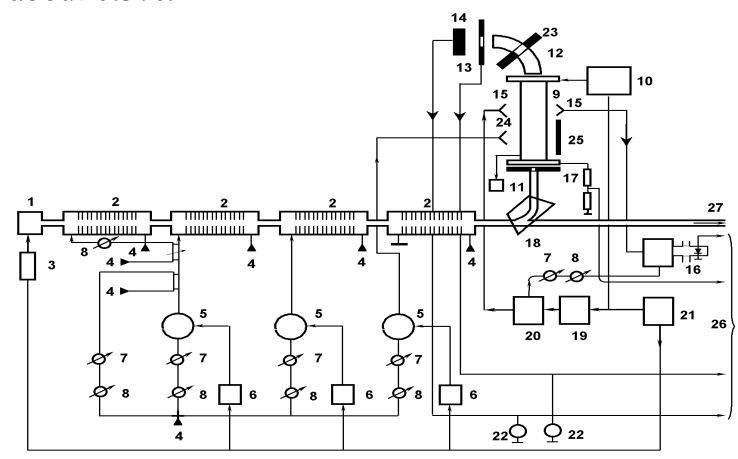

Figure 1: Experimental scheme of the device: (1) electron source, (2) diaphragmatic waveguide, (3) electron source modulator, (4) matched microwave load, (5) high-power klystron (P puls »20 MW), (6) klystron modulator, (7) attenuator, (8) phase shifter, (9) plasma chamber, (10) modulator supplying the plasma chamber, (11) forevacuum pump, (12) magnetic analyzer, (13) collimator, (14) Faraday cup, (15) emitting and receiving antennas, (16) radio interferometer, (17) load resistor, (18) beam-deflecting magnet, (19) microwave oscillator, (20) microwave amplifier, (21) synchronization unit, (22) microammeter, (23) additional solenoid, (24) antenna emitting a high-power microwave signal, (25) reflector, (26) signals fed to an oscilloscope, and (27) electron beam escaping from the linac.

\footnotetext{
oksuzyan@moon.yerphi.am
} 
Figures 2 and 3 show the electron energy spectra of a beam with the maximum current at before and after interaction with a plasma. The energy spectra of the beam electrons are incomplete (the wings of the spectrum are absent) because, at the exit from the interaction chamber, the average electron beam current was too low $\left(\mathrm{I}_{\mathrm{b}}<0.1\right.$ $\mu \mathrm{A})$ to be measured reliably when the field of the magnetic analyzer changed substantially. For this reason, the measurements were aimed at revealing the largest displacement of the energy spectrum toward high energies. In a plasma with a density of about $\sim 4 \cdot 10^{11} \mathrm{~cm}^{-3}$, the increment in the energy of the beam electrons injected at energies of about $50 \mathrm{MeV}$ was found to be $0.6 \mathrm{MeV}$. For an injection energy of about $20 \mathrm{MeV}$ and a plasma density of about $\sim 10^{11} \mathrm{~cm}^{-3}$ (which corresponds to the electron plasma frequency close to the modulation frequency of the beam), the peak in the energy spectrum was observed to be displaced toward higher energies by approximately $0.2 \mathrm{MeV}$. This result agrees with the conclusion drawn by paper [6]: the higher the energy of the injected electron beam, the higher the beam--plasma interaction efficiency. The second series of experiments was carried out with a plasma created by a

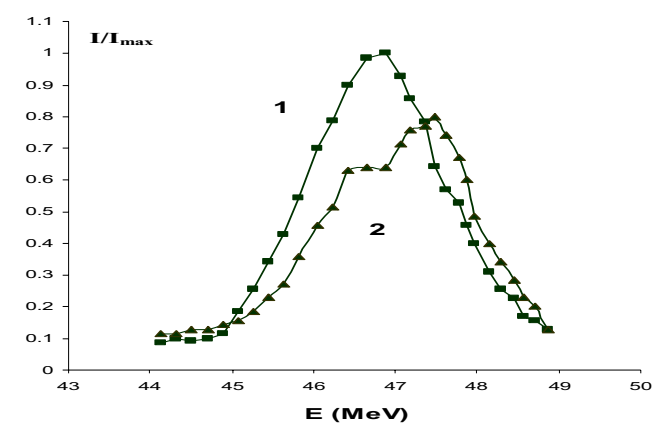

Figure 2: Energy spectra of the beam electrons (1) in the absence of a plasma and (2) in the presence of a plasma of density $\mathrm{n}_{\mathrm{p}} \sim 4 \cdot 10^{11} \mathrm{~cm}^{-3}$.

microwave pulse in an open resonator (Fig.1). The electric field vector $\mathrm{E}$ of the microwave field was oriented along the plasma cham- ber, i.e., in the propagation direction of the electron bunches. In order for the plasma to be produced most intensity, the distance $d(d \sim \lambda=$ $10.72 \mathrm{~cm}$, where 1 is the radiation wavelength) between reflector (25) and the emitting open end of the waveguide was chosen in such a way that the antinode of the standing wave of microwave oscillations occurred at the symmetry axis of the plasma chamber. Microwave radiation was fed into the plasma chamber from the accelerator microwave power supply system (klystron 5). As a result, the plasma was created by the same microwave field that was used to form electron bunches and to accelerate them to an energy of $20 \mathrm{MeV}$. Consequently, the interaction of electron bunches with a plasma whose density $\mathrm{n} \mathrm{p}$ was constant during the microwave pulse was coherent in character. This is illustrated in Fig. 4, which shows the current of the electrons accelerated to an energy of $20 \mathrm{MeV}$ after they passed through the entire plasma region versus the microwave field phase, which was biased with respect to the phase of a periodic sequence of electron bunches. Unfortunately, with a klystron whose nominal microwave power was about $20 \mathrm{MW}$, the maximum microwave power did not exceed $\sim 100 \mathrm{~kW}$ because of the low electric strength of the waveguide. Also, the intense scattering of electromagnetic waves in the open resonator did not allow us to concentrate the microwave energy and to achieve strong microwave fields at the axis of the plasma chamber. For this reason, the plasma density in our experiments was too low to achieve resonance interaction conditions. That is why our measurements of the electron energy spectrum at different beam currents (see Fig. 4) revealed no significant difference in the spectral distribution of the beam electrons. By increasing the plasma density at the expense of the constant voltage component of a pulsed modulator while simultaneously feeding microwave and high voltage pulses, we observed that the electron beam current became completely effect can find applications, in particular, in fusion

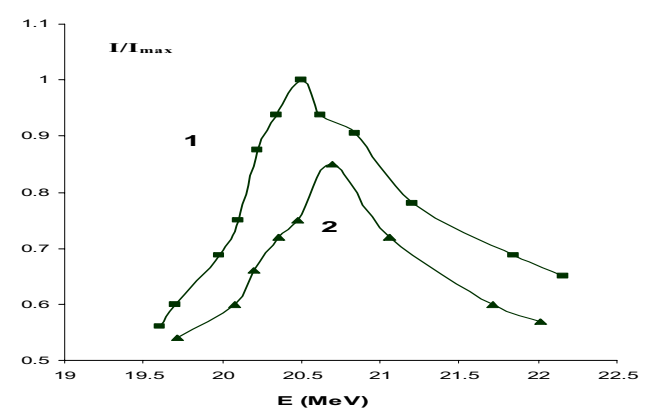

Figure 3: Energy spectra of the beam electrons (1) in the absence of a plasma and (2) in the presence of a plasma of density $\mathrm{n}_{\mathrm{p}} \sim 10^{11} \mathrm{~cm}^{-3}$.

independent of the wave phase. This effect can be explained as being due to the high voltages and high currents generated by a pulsed modulator: after switching on the modulator, the microwave discharge was observed to decay, because the constant component of the plasma current destroyed the spatial periodicity of the distribution of the electron plasma density; as a result, the electron beam current became independent of the wave phase. This phenomenon can be used to obtain indirect estimates of the amplitude of the microwave field generated in the plasma. Under the coherent interaction conditions (Fig. 4), the fraction of the scattered (absorbed) beam current in a microwave field with a comparatively small amplitude $(<0.05$ of the amplitude of the microwave field that was used to accelerate the beam) amounted to $70--80 \%$. In this case, the relative increase in the current can attain $10 \%$ per degree of the phase of the microwave field. This devices in which the plasma is heated by high-current relativistic electron beams [3]. In closed electrodynamic systems, varying the amplitude of the microwave field and biasing its phase with respect to the phase of a periodic sequence of electron bunches provides more efficient control of the generation of potential waves without energy losses due to the excitation of radiation at 


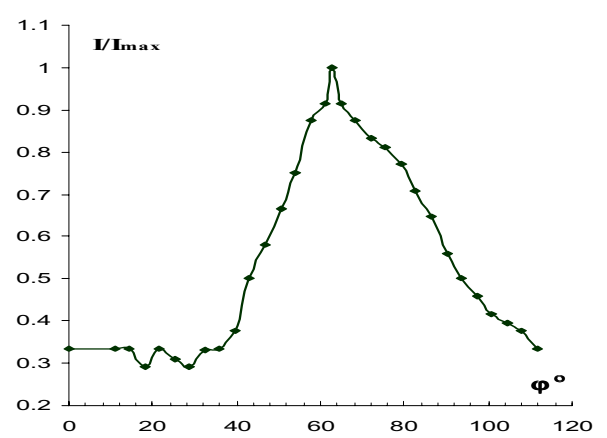

Figure 4: Current of the electrons of an injected $20 \mathrm{MeV}$ beam that pass through the plasma vs the phase of the microwave field. The plasma was produced by a microwave pulse with a power of about $\mathrm{P}_{\text {puls }} \sim 100 \mathrm{~kW}$. The phase of the microwave field was biased with respect to the phase of a periodic sequence of electron bunches that were formed and accelerated to $20 \mathrm{MeV}$ by the same microwave pulse in the accelerating sections of the linac.

the combination frequencies. The strong coupling of the phase of the oscillations at the combination frequencies to the phase of the original microwave field can provide optimum interaction conditions (the polarization losses change sign $[12,13])$ for raising the wakefield amplitude. Also, under the coherent interaction conditions, the so-called zero method makes it possible to find the relation between the beam and plasma parameters, i.e., to estimate the beam-- plasma interaction efficiency. In our experiments, a $20-\mathrm{MeV}$ electron beam was completely scattered (absorbed) over a distance of about 10--20 cm (with allowance for the nonuniform character of ionization) in the plasma chamber, thereby providing evidence of the possible onset of strong microwave fields (both transverse and longitudinal). Hence, the plasma experienced both internal and external forces exerted by the electron beam and microwave pulse, respectively. The revealed strong coherent dependence of the current of a relativistic electron beam on the phase of the microwave field holds promise for increasing the electron acceleration rate through the direct excitation of high-power parametric oscillations [14]. The pump frequency w pump (the same as the frequency of the plasma-producing microwave field) can generally be higher than (or equal to) $\mathrm{nw}_{\mathrm{m}}$, where $\mathrm{w}_{\mathrm{m}}$ is the frequency of the accelerating field or, equivalently, the modulation frequency of an electron beam. This relationship between the frequencies can play an important role at high plasma densities. It is well known that the most stable parametric resonance occurs under the condition $\mathrm{w}_{\text {pump }}=2 \mathrm{w}_{\mathrm{m}}$. This condition is especially important because the difference combination frequency is equal to the frequency of the accelerating field, $\mathrm{w}_{\text {comb }}=\mathrm{w}_{\text {pump }}-\mathrm{w}_{\mathrm{m}}=\mathrm{w}_{\mathrm{m}}$ [15]. The plasma excitation by high- power microwaves at the second harmonic of the accelerating field can be achieved through frequency multiplication followed by the amplification of the input signal power. Controlling the amplitude of the microwave field under the coherent interaction conditions makes it possible to equate the frequencies, $\mathrm{w}_{\mathrm{m}}=\mathrm{w}_{\mathrm{p}}$ (where $\mathrm{w}_{\mathrm{p}}$ is the plasma frequency), i.e., to achieve the resonant interaction conditions. For higher plasma densities (e.g., those corresponding to the optical range), the coherent interaction condition $\mathrm{w}_{\text {pump }}=$ $2 \mathrm{w}_{\mathrm{m}}$ can be achieved by using the free electron laser (FEL) scheme [3, 16]. Hence, in the three-wave approximation, the energy of Langmuir oscillations of the plasma, the wakefield of a periodic sequence of electron bunches, and the pump field can (in case of the corresponding phasing of the waves)act to enhance the wake potential and thus to increase the electron acceleration rate.

\section{REFERENCES}

[1] Ya. B. Fainberg, Proc. Symp. CERN 1, 84 (1956).

[2] Ya. B. Fainberg, Fiz. Plazmy 13, 607 (1987) [Sov.J. Plasma Phys. 13, 350 (1987)].

[3] Ya. B. Fainberg, Fiz. Plazmy 20, 613 (1994) [Plasma Phys. Rep. 20, 549 (1994)].

[4] Ya. B. Fainberg, Fiz. Plazmy 23, 275 (1997) [Plasma Phys. Rep. 23, 251 (1997)].

[5] A. Ts. Amatuni, M. R. Magometov, E. V. Sekhposyan and S. S. Elbakyan, Fiz. Plazmy 5, 85 (1979) [Sov. J. Plasma Phys. 5, 49 (1979)].

[6] A. Ts. Amatuni, E. V. Sekhposyan, and S. S. Elbakyan, Fiz. Plazmy 12, 1145 (1986) [Sov. J. Plasma Phys. 12, 662 (1986)].

[7] A. K. Berezin, Ya. B. Fainberg, L. I. Bolotin, et al., Zh. Eksp. Teor. Fiz. 63, 861 (1972) [Sov. Phys. JETP 36, 453 (1973)].

[8] P. Chen, J. M. Dawson, R. W. Huff, and T. Katsouleas, Phys. Rev. Lett. 54, 693 (1985).

[9] J. B. Rosenzweig, D. B. Cline, B. Cole, et al., Phys. Rev. Lett. 61, 98 (1988).

[10] A. K. Berezin, Ya. B. Fainberg, V. A. Kiselev, et al., Fiz. Plazmy 20, 663 (1994) [Plasma Phys. Rep. 20, 596 (1994)].

[11] Ya. B. Fainberg, N. I. Aizatskii, V. A. Balakirev, et al., in Proceedings of the XV International Workshop on Charged-Particle Accelerators, Alushta, 1997.

[12] Yu. M. Aliev, L. M. Gorbunov, and R. R. Ramazashvili, Zh. Eksp. Teor. Fiz. 61, 1477 (1971) [Sov. Phys. JETP 34, 785 (1972)].

[13] E. A. Akopyan and G. G. Matevosyan, Fiz. Plazmy 10, 648 (1984) [Sov. J. Plasma Phys. 10, 378 (1984)].

[14] V. P. Silin, Parametric Influence of High Power Radiation on Plasma (Nauka, Moscow, 1973).

[15] V. M. Lopukhin and A. S. Roshal', Electron-beam Para-metric Amplifiers (Sov. Radio, Moscow, 1968).

[16] V. I. Miroshnichenko, Ya. B. Fainberg and A. E. Volkov, Fiz. Plazmy 20, 620 (1994) [Plasma Phys. Rep. 20, 555 (1994)]. 